\title{
Understanding the Algebraic and Logical Structure of Speech Perception
}

\author{
Kalina A. Misiolek ${ }^{1}$, Robert M. Worth ${ }^{2,3}$, Leonid L. Rubchinsky ${ }^{3,4}$ \\ ${ }^{1}$ Indiana University School of Medicine, ${ }^{2}$ Indiana University School of Medicine, \\ Department of Neurological Surgery, ${ }^{3}$ Indiana University-Purdue University \\ Indianapolis, Department of Mathematical Sciences, ${ }^{4}$ Indiana University School \\ of Medicine, Stark Neurosciences Research Institute
}

Background: Although subject to much variation, the anatomy of language comprehension has become increasingly clear with the advent of fMRI; however, the steps of speech comprehension remain elusive. There are two main theories of language processing - hierarchical and sequential (or probabilistic). According to the hierarchical theory, sentences are broken down (e.g. sentence to words to syllables to phonemes) and then reconstructed while syntactic and semantic meanings are attached. Sequential theory suggests the use of "word-level statistics" and n-gram-type models to predict sequences of word meanings. ${ }^{1}$ Although evidence suggests both models play some role, as a starting point, many comprehension models focus on hierarchical theory, and many of those in turn rely on neural networks. However, in various ways, these models fall short of explaining how the brain can biologically carry out all the steps.

Methods: We attempt to create a hierarchical model of speech comprehension using linear logic (or a related logic) or Category Theory, with the hope that such an approach may be able to explain the process more naturally. We focus on the second half of comprehension (i.e. the reconstruction) to make use of existing neuronal logic gate models. ${ }^{2}$ The goal is to construct a linear logic model or to create categories and associated functors that could explain hierarchical linguistic processing and many neurolinguistic study results.

Potential Impact: Although this model would only account for hierarchical linguistic processing, it would be a huge step forward in understanding how our brain processes speech - and possibly other inputs - at the level of neuron bundles.

\section{References:}

1. Frank, S. L., Christiansen, M. H. (2018). Hierarchical and sequential processing of language. Language, Cognition and Neuroscience, 1-6. doi:10.1080/23273798.2018.1424347

2. Goldental, A., Guberman, S., Vardi, R., \& Kanter, I. (2014). A computational paradigm for dynamic logic-gates in neuronal activity. Frontiers in Computational Neuroscience, 8. doi:10.3389/fncom.2014.00052 